\title{
A Theory of Universal Learning
}

\author{
Olivier Bousquet \\ Google, Brain Team \\ Zürich, Switzerland \\ obousquet@google.com
}

\author{
Steve Hanneke \\ Toyota Technological Inst. at Chicago \\ Chicago, Illinois, USA \\ steve.hanneke@gmail.com
}

\author{
Shay Moran* \\ Technion \& Google Research \\ Haifa, Israel \\ smoran@technion.ac.il
}

\author{
Ramon van Handel \\ PACM, Princeton University \\ Princeton, New Jersey, USA \\ rvan@math.princeton.edu
}

\author{
Amir Yehudayoff \\ Department of Mathematics, \\ Technion \\ Haifa, Israel \\ amir.yehudayoff@gmail.com
}

\begin{abstract}
How quickly can a given class of concepts be learned from examples? It is common to measure the performance of a supervised machine learning algorithm by plotting its "learning curve", that is, the decay of the error rate as a function of the number of training examples. However, the classical theoretical framework for understanding learnability, the PAC model of Vapnik-Chervonenkis and Valiant, does not explain the behavior of learning curves: the distribution-free PAC model of learning can only bound the upper envelope of the learning curves over all possible data distributions. This does not match the practice of machine learning, where the data source is typically fixed in any given scenario, while the learner may choose the number of training examples on the basis of factors such as computational resources and desired accuracy.

In this paper, we study an alternative learning model that better captures such practical aspects of machine learning, but still gives rise to a complete theory of the learnable in the spirit of the PAC model. More precisely, we consider the problem of universal learning, which aims to understand the performance of learning algorithms on every data distribution, but without requiring uniformity over the distribution. The main result of this paper is a remarkable trichotomy: there are only three possible rates of universal learning. More precisely, we show that the learning curves of any given concept class decay either at an exponential, linear, or arbitrarily slow rates. Moreover, each of these cases is completely characterized by appropriate combinatorial parameters, and we exhibit optimal learning algorithms that achieve the best possible rate in each case.

For concreteness, we consider in this paper only the realizable case, though analogous results are expected to extend to more general learning scenarios.
\end{abstract}

${ }^{*}$ Robert J. Shillman Fellow. Research supported in part by the Israel Science Foundation (grant No. 1225/20), by an Azrieli Faculty Fellowship, and by a grant from the United States - Israel Binational Science Foundation (BSF).

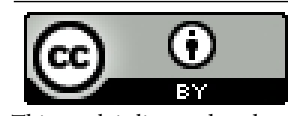

This work is licensed under a Creative Commons Attribution International 4.0 License. STOC '21, fune 21-25, 2021, Virtual, Italy

(c) 2021 Copyright held by the owner/author(s).

ACM ISBN 978-1-4503-8053-9/21/06.

https://doi.org/10.1145/3406325.3451087

\section{CCS CONCEPTS}

- Theory of computation $\rightarrow$ Sample complexity and generalization bounds.

\section{KEYWORDS}

Statistical Learning, Distribution-Dependent Learning Rates, Online Learning

ACM Reference Format:

Olivier Bousquet, Steve Hanneke, Shay Moran, Ramon van Handel, and Amir Yehudayoff. 2021. A Theory of Universal Learning. In Proceedings of the 53rd Annual ACM SIGACT Symposium on Theory of Computing (STOC '21), June 21-25, 2021, Virtual, Italy. ACM, New York, NY, USA, 10 pages. https: //doi.org/10.1145/3406325.3451087

\section{INTRODUCTION}

In supervised machine learning, a learning algorithm is presented with labeled examples of a concept, and the objective is to output a classifier which correctly classifies most future examples from the same source. Supervised learning has been successfully applied in a vast number of scenarios, such as image classification and natural language processing. In any given scenario, it is common to consider the performance of an algorithm by plotting its "learning curve", that is, the error rate (measured on held-out data) as a function of the number of training examples $n$. A learning algorithm is considered successful if the learning curve approaches zero as $n \rightarrow \infty$, and the difficulty of the learning task is reflected by the rate at which this curve approaches zero. One of the main goals of learning theory is to predict what learning rates are achievable in a given learning task.

To this end, the gold standard of learning theory is the celebrated PAC model (Probably Approximately Correct) defined by Vapnik and Chervonenkis [27] and Valiant [24]. As will be recalled below, the PAC model aims to explain the best worst-case learning rate, over all data distributions that are consistent with a given concept class, that is achievable by a learning algorithm. The fundamental result in this theory exhibits a striking dichotomy: a given learning problem either has a linear worst-case learning rate (i.e., $n^{-1}$ ), or is not learnable at all in this sense. These two cases are characterized by a fundamental combinatorial parameter of a learning problem: the $V C$ (Vapnik-Chervonenkis) dimension. Moreover, in the learnable case, PAC theory provides optimal learning algorithms that achieve the linear worst-case rate. 
While it gives rise to a clean and compelling mathematical picture, one may argue that the PAC model fails to capture at a fundamental level the true behavior of many practical learning problems. A key criticism of the PAC model is that the distributionindependent definition of learnability is too pessimistic to explain practical machine learning: real-world data is rarely worst-case, and experiments show that practical learning rates can be $m u c h$ faster than is predicted by PAC theory [7, 8]. It therefore appears that the worst-case nature of the PAC model hides key features that are observed in practical learning problems. These considerations motivate the search for alternative learning models that better capture the practice of machine learning, but still give rise to a canonical mathematical theory of learning rates. Moreover, given a theoretical framework capable of expressing these faster learning rates, we can then design new learning strategies to fully exploit this possibility.

The aim of this paper is to put forward one such theory. In the learning model considered here, we will investigate asymptotic rates of convergence of distribution-dependent bounds on the error of a learning algorithm, holding universally for all distributions consistent with a given concept class. Despite that this is a much weaker (and therefore arguably more realistic) notion, we will nonetheless prove that any learning problem can only exhibit one of three possible universal rates: exponential, linear, and arbitrarily slow. Each of these three cases will be fully characterized by means of combinatorial parameters (the nonexistence of certain infinite trees), and we will exhibit optimal learning algorithms that achieve these rates (based on the theory of infinite games).

Organization. This extended abstract is organized as follows. In the remainder of this section we define the learning setting, state our main result, present some basic examples, and survey related work. In Section 2 we present more advanced examples, which manifest the landscape of universal learning, and how it relates to the landscape of PAC learning. The proofs can be found in the full version on arXiv [6].

\subsection{The Basic Learning Problem}

Throughout this paper we will be concerned with the following classical learning problem. A classification problem is defined by a distribution $P$ over labelled examples $(x, y) \in \mathcal{X} \times\{0,1\}$. The learner does not know $P$, but is able to collect a sample of $n$ i.i.d. examples from $P$. She uses these examples to build a classifier $\hat{h}_{n}: \mathcal{X} \rightarrow\{0,1\}$. The objective of the learner is to achieve small error:

$$
\operatorname{er}\left(\hat{h}_{n}\right):=P\left\{(x, y): \hat{h}_{n}(x) \neq y\right\} .
$$

While the data distribution $P$ is unknown to the learner, any informative a priori theory of learning must be expressed in terms of some properties of, or restrictions on, $P$. Following the PAC model, we introduce such a restriction by way of an additional component, namely a concept class $\mathcal{H} \subseteq\{0,1\}^{\mathcal{X}}$ of classifiers. The concept class $\mathcal{H}$ allows the analyst to state assumptions about $P$. The simplest such assumption is that $P$ is realizable:

$$
\inf _{h \in \mathcal{H}} \operatorname{er}(h)=0,
$$

that is, $\mathcal{H}$ contains hypotheses with arbitrarily small error. We will focus on the realizable setting throughout this paper, as it already

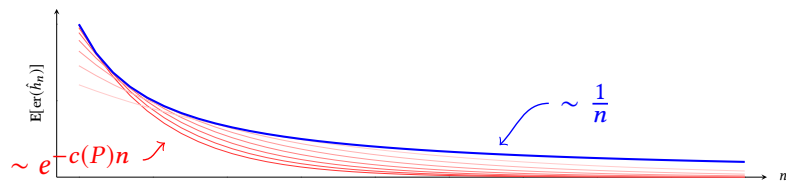

Figure 1: Illustration of the difference between universal and uniform rates. Each red curve shows exponential decay of the error for a different data distribution $P$; but the PAC rate only captures the pointwise supremum of these curves (blue curve) which decays linearly at best.

requires substantial new ideas and provides a clean platform to demonstrate them. We believe that the ideas of this paper can be extended to more general noisy/agnostic settings, and leave this direction to be explored in future work.

In the present context, the aim of learning theory is to provide tools for understanding the best possible rates of convergence of $\mathbf{E}\left[\operatorname{er}\left(\hat{h}_{n}\right)\right]$ to zero as the sample size $n$ grows to $\infty$. This rate depends on the quality of the learning algorithm, and on the complexity of the concept class $\mathcal{H}$. The more complex $\mathcal{H}$ is, the less information the learner has about $P$, and thus the slower the convergence.

\subsection{Uniform and Universal Rates}

The classical formalization of the problem of learning in statistical learning theory is given by the PAC model, which adopts a minimax perspective. More precisely, let us denote by $\operatorname{RE}(\mathcal{H})$ the family of distributions $P$ for which the concept class $\mathcal{H}$ is realizable. Then the fundamental result of PAC learning theory states that $[11,16,27]$

$$
\inf _{\hat{h}_{n}} \sup _{P \in \operatorname{RE}(\mathcal{H})} \operatorname{E}\left[\operatorname{er}\left(\hat{h}_{n}\right)\right] \asymp \min \left(\frac{\operatorname{vc}(\mathcal{H})}{n}, 1\right),
$$

where $\operatorname{vc}(\mathcal{H})$ is the $V C$ dimension of $\mathcal{H}$. In other words, PAC learning theory is concerned with the best worst-case error over all realizable distributions, that can be achieved by means of a learning algorithm $\hat{h}_{n}$. The above result immediately implies a fundamental dichotomy for these uniform rates: every concept class $\mathcal{H}$ has a uniform rate that is either linear $\frac{c}{n}$ or bounded away from zero, depending on the finiteness of the combinatorial parameter $\operatorname{vc}(\mathcal{H})$.

The uniformity over $P$ in the PAC model is very pessimistic, however, as it allows the worst-case distribution to change with the sample size. This arguably does not reflect the practice of machine learning: in a given learning scenario, the data generating mechanism $P$ is fixed, while the learner is allowed to collect an arbitrary amount of data (depending on factors such as the desired accuracy and the available computational resources). Experiments show that the rate at which the error decays for any given $P$ can be much faster than is suggested by PAC theory [7, 8]: for example, it is possible that the learning curve decays exponentially for every $P$. Such rates cannot be explained by the PAC model, which can only capture the upper envelope of the learning curves over all realizable $P$, as is illustrated in Figure 1.

Furthermore, one may argue that it is really the learning curve for given $P$, rather than the PAC error bound, that is observed in practice. Indeed, the customary approach to estimate the performance of an algorithm is to measure its empirical learning rate, 
that is, to train it on several training sets of increasing sizes (obtained from the same data source) and to measure the test error of each of the obtained classifiers. In contrast, to observe the PAC rate, one would have to repeat the above measurements for many different data distributions, and then discard all this data except for the worst-case error over all considered distributions. From this perspective, it is inevitable that the PAC model may fail to reveal the "true" empirical behavior of learning algorithms. More refined theoretical results have been obtained on a case-by-case basis in various practical situations: for example, under margin assumptions, some works established exponentially fast learning rates for popular algorithms such as stochastic gradient decent and kernel methods $[2,17,19,21]$. Such results rely on additional modelling assumptions, however, and do not provide a fundamental theory of the learnable in the spirit of PAC learning.

Our aim in this paper is to propose a mathematical theory that is able to capture some of the above features of practical learning systems, yet provides a complete characterization of achievable learning rates for general learning tasks. Instead of considering uniform learning rates as in the PAC model, we consider instead the problem of universal learning. The term universal means that a given property (such as consistency or rate) holds for every realizable distribution $P$, but not uniformly over all distributions. For example, a class $\mathcal{H}$ is universally learnable at rate $R$ if the following holds:

$$
\exists \hat{h}_{n} \text { s.t. } \forall P \in \operatorname{RE}(\mathcal{H}), \exists C, c>0 \text { s.t. }(\forall n): \mathrm{E}\left[\operatorname{er}\left(\hat{h}_{n}\right)\right] \leq C R(c n) .
$$

The crucial difference between this formulation and the PAC model is that here the constants $C, c$ are allowed to depend on $P$ : thus universal learning is able to capture distribution-dependent learning curves for a given learning task. For example, the illustration in Figure 1 suggests that it is perfectly possible for a concept class $\mathcal{H}$ to be universally learnable at an exponential rate, even though its uniform learning rate is only linear. In fact, we will see that there is little connection between universal and uniform learning rates (as is illustrated in Figure 4 of section 2): a given problem may even be universally learnable at an exponential rate while it is not learnable at all in the PAC sense. These two models of learning reveal fundamentally different features of a given learning problem.

The fundamental question that we pose in this paper is:

Question. Given a class $\mathcal{H}$, what is the fastest rate at which $\mathcal{H}$ can be universally learned?

We provide a complete answer to this question, characterize the achievable rates by means of combinatorial parameters, and exhibit learning algorithms that achieve these rates. The universal learning model therefore gives rise to a theory of learning that fully complements the classical PAC theory.

\subsection{Basic Examples}

Before we proceed to the statement of our main results, we aim to develop some initial intuition for what universal learning rates are achievable. To this end, we briefly discuss three basic examples.

Example 1.1. Any finite class $\mathcal{H}$ is universally learnable at an exponential rate [22]. Indeed, let $\varepsilon$ be the minimal error er $(h)$ among all classifiers $h \in \mathcal{H}$ with positive error $\operatorname{er}(h)>0$. By the union bound, the probability that there exists a classifier with positive error that correctly classifies all $n$ training data points is bounded by $|\mathcal{H}|(1-\varepsilon)^{n}$. Thus a learning rule that outputs any $\hat{h}_{n} \in \mathcal{H}$ that correctly classifies the training data satisfies $\operatorname{E}\left[\operatorname{er}\left(\hat{h}_{n}\right)\right] \leq C e^{-c n}$, where $C, c>0$ depend on $\mathcal{H}, P$. It is easily seen that this is the best possible: as long as $\mathcal{H}$ contains at least three functions, a learning curve cannot decay faster than exponentially (see Theorem 1.9 below).

Example 1.2. The class $\mathcal{H}=\left\{h_{t}: t \in \mathbb{R}\right\}$ of threshold classifiers on the real line $h_{t}(x)=1_{x \geq t}$ is universally learnable at a linear rate. That a linear rate can be achieved already follows in this case from PAC theory, as $\mathcal{H}$ is a VC class. However, in this example, a linear rate is the best possible even in the universal setting: for any learning algorithm, there is a realizable distribution $P$ whose learning curve decays no faster than a linear rate [22].

Example 1.3. The class $\mathcal{H}$ of all measurable functions on a space $\mathcal{X}$ is universally learnable under mild conditions [15, 23]: that is, there exists a learning algorithm $\hat{h}_{n}$ that ensures $\mathrm{E}\left[\operatorname{er}\left(\hat{h}_{n}\right)\right] \rightarrow 0$ as $n \rightarrow \infty$ for every realizable distribution $P$. However, there can be no universal guarantee on the learning rate [9]. That is, for any learning algorithm $\hat{h}_{n}$ and any function $R(n)$ that converges to zero arbitrarily slowly, there exists a realizable distribution $P$ such that $\mathbf{E}\left[\operatorname{er}\left(\hat{h}_{n}\right)\right] \geq R(n)$ infinitely often.

The three examples above reveal that there are at least three possible universal learning rates. Remarkably, we find that these are the only possibilities. That is, every nontrivial class $\mathcal{H}$ is either universally learnable at an exponential rate (but not faster), or is universally learnable at a linear rate (but not faster), or is universally learnable but necessarily with arbitrarily slow rates.

\subsection{Main Results}

We now summarize the key definitions and main results of the paper. (We refer to the Appendix of the full paper [6] for the relevant terminology on Polish spaces and measurability.)

To specify the learning problem, we specify a domain $X$ and a concept class $\mathcal{H} \subseteq\{0,1\}^{X}$. We will henceforth assume that $\mathcal{X}$ is a Polish space (for example, a Euclidean space, or any countable set) and that $\mathcal{H}$ satisfies a minimal measurability assumption specified in the full paper [6].

A classifier is a universally measurable function $h: \mathcal{X} \rightarrow\{0,1\}$. Given a probability distribution $P$ on $X \times\{0,1\}$, the error rate of a classifier $h$ is defined as

$$
\operatorname{er}(h)=\operatorname{er}_{P}(h):=P\{(x, y): h(x) \neq y\} .
$$

The distribution $P$ is called realizable if $\inf _{h \in \mathcal{H}} \operatorname{er}(h)=0$.

A learning algorithm is a sequence of universally measurable functions ${ }^{1}$

$$
H_{n}:(X \times\{0,1\})^{n} \times X \rightarrow\{0,1\}, \quad n \in \mathbb{N} .
$$

The input data to the learning algorithm is a sequence of independent $P$-distributed pairs $\left(X_{i}, Y_{i}\right)$. When acting on this input data,

\footnotetext{
${ }^{1}$ For simplicity of exposition, we have stated a definition corresponding to deterministic algorithms, to avoid the notational inconvenience required to formally define randomized algorithms in this context. Our results remain valid when allowing randomized algorithms as well: all algorithms we construct throughout this paper are deterministic, and all lower bounds we prove also hold for randomized algorithms.
} 
the learning algorithm outputs the data-dependent classifiers

$$
\hat{h}_{n}(x):=H_{n}\left(\left(X_{1}, Y_{1}\right), \ldots,\left(X_{n}, Y_{n}\right), x\right) .
$$

The objective in the design of a learning algorithm is that the expected error rate $\mathbf{E}\left[\operatorname{er}\left(\hat{h}_{n}\right)\right]$ of the output concept decays as rapidly as possible as a function of $n$.

The aim of this paper is to characterize what rates of convergence of $\mathbf{E}\left[\operatorname{er}\left(\hat{h}_{n}\right)\right]$ are achievable. The following definition formalizes this notion of achievable rate in the universal learning model.

Definition 1.4. Let $\mathcal{H}$ be a concept class, and let $R: \mathbb{N} \rightarrow[0,1]$ with $R(n) \rightarrow 0$ be a rate function.

- $\mathcal{H}$ is learnable at rate $R$ if there is a learning algorithm $\hat{h}_{n}$ such that for every realizable distribution $P$, there exist $C, c>0$ for which $\mathrm{E}\left[\operatorname{er}\left(\hat{h}_{n}\right)\right] \leq C R(c n)$ for all $n$.

- $\mathcal{H}$ is not learnable at rate faster than $R$ if for every learning algorithm $\hat{h}_{n}$, there exists a realizable distribution $P$ and $C, c>0$ for which $\mathrm{E}\left[\operatorname{er}\left(\hat{h}_{n}\right)\right] \geq C R(c n)$ for infinitely many $n$

- $\mathcal{H}$ is learnable with optimal rate $R$ if $\mathcal{H}$ is learnable at rate $R$ and $\mathcal{H}$ is not learnable faster than $R$.

- $\mathcal{H}$ requires arbitrarily slow rates if, for every $R(n) \rightarrow 0$, $\mathcal{H}$ is not learnable faster than $R$.

Let us emphasize that, unlike in the PAC model, every concept class $\mathcal{H}$ is universally learnable in the sense that there exist learning algorithms such that $\mathrm{E}\left[\operatorname{er}\left(\hat{h}_{n}\right)\right] \rightarrow 0$ for all realizable $P$; see Example 1.3 above. However, a concept class may nonetheless require arbitrarily slow rates, in which case it is impossible for the learner to predict how fast this convergence will take place.

Remark 1.5. While this is not assumed in the above definition, our lower bound results will in fact prove a stronger claim: namely, that when a given concept class $\mathcal{H}$ is not learnable at rate faster than $R$, the corresponding constants $C, c>0$ in the lower bound can be specified as universal constants, that is, they are independent of the learning algorithm $\hat{h}_{n}$ and concept class $\mathcal{H}$. This is sometimes referred to as a strong minimax lower bound [1].

The following theorem is one of the main results of this work. It expresses a fundamental trichotomy: there are exactly three possibilities for optimal learning rates. ${ }^{2}$

Theorem 1.6. For every concept class $\mathcal{H}$ with $|\mathcal{H}| \geq 3$, exactly one of the following holds.

- $\mathcal{H}$ is learnable with optimal rate $e^{-n}$.

- $\mathcal{H}$ is learnable with optimal rate $\frac{1}{n}$.

- $\mathcal{H}$ requires arbitrarily slow rates.

A second main result of this work provides a detailed description of which of these three cases any given concept class $\mathcal{H}$ satisfies, by specifying complexity measures to distinguish the cases. We begin with the following definition, which is illustrated in Figure 2. Henceforth we define the prefix $\mathbf{y}_{\leq k}:=\left(y_{1}, \ldots, y_{k}\right)$ for any sequence $\mathbf{y}=\left(y_{1}, y_{2}, \ldots\right)$.

${ }^{2}$ The restriction $|\mathcal{H}| \geq 3$ rules out two degenerate cases: if $|\mathcal{H}|=1$ or if $\mathcal{H}=\{h, 1-$ $h\}$, then $\operatorname{er}\left(\hat{h}_{n}\right)=0$ is trivially achievable for all $n$. If $|\mathcal{H}|=2$ but $\mathcal{H} \neq\{h, 1-h\}$, then $\mathcal{H}$ is learnable with optimal rate $e^{-n}$ by Example 1.1.

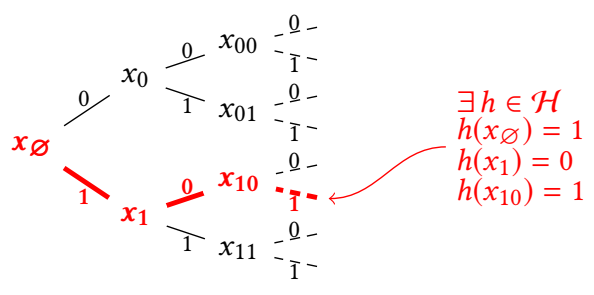

Figure 2: A Littlestone tree of depth 3. Every branch is consistent with a concept $h \in \mathcal{H}$. This is illustrated here for one of the branches.

Definition 1.7. A Littlestone tree for $\mathcal{H}$ is a complete binary tree of depth $d \leq \infty$ whose internal nodes are labelled by $\mathcal{X}$, and whose two edges connecting a node to its children are labelled 0 and 1 , such that every finite path emanating from the root is consistent with a concept $h \in \mathcal{H}$.

More precisely, a Littlestone tree is a collection

$$
\left\{x_{\mathbf{u}}: 0 \leq k<d, \mathbf{u} \in\{0,1\}^{k}\right\} \subseteq \mathcal{X}
$$

such that for every y $\in\{0,1\}^{d}$ and $n<d$, there exists $h \in \mathcal{H}$ so that $h\left(x_{\mathbf{y}_{\leq k}}\right)=y_{k+1}$ for $0 \leq k \leq n$. We say $\mathcal{H}$ has an infinite Littlestone tree if there is a Littlestone tree for $\mathcal{H}$ of depth $d=\infty$.

The above notion is closely related to the Littlestone dimension, a fundamentally important quantity in online learning. A concept class $\mathcal{H}$ has Littlestone dimension $d$ if it has a Littlestone tree of depth $d$ but not of depth $d+1$. When this is the case, classical online learning theory yields a learning algorithm that makes at most $d$ mistakes in classifying any adversarial (as opposed to random) realizable sequence of examples. Along the way to our main results, we will extend the theory of online learning to the following setting: we show in the full paper [6] that the nonexistence of an infinite Littlestone tree characterizes the existence of an algorithm that guarantees a finite (but not necessarily uniformly bounded) number of mistakes for every realizable sequence of examples. Let us emphasize that having an infinite Littlestone tree is not the same as having an unbounded Littlestone dimension: the latter can happen due to existence of finite Littlestone trees of arbitrarily large depth, which does not imply the existence of any single tree of infinite depth.

Next we introduce a new type of complexity structure, which we term a $V C$-Littlestone tree. It represents a combination of the structures underlying Littlestone dimension and VC dimension. Though the definition may appear a bit complicated, the intuition is quite simple (see Figure 3).

Definition 1.8. A VCL tree for $\mathcal{H}$ of depth $d \leq \infty$ is a collection

$$
\left\{x_{\mathbf{u}} \in \mathcal{X}^{k+1}: 0 \leq k<d, \mathbf{u} \in\{0,1\}^{1} \times\{0,1\}^{2} \times \cdots \times\{0,1\}^{k}\right\}
$$

such that for every $n<d$ and $\mathbf{y} \in\{0,1\}^{1} \times \cdots \times\{0,1\}^{n+1}$, there exists a concept $h \in \mathcal{H}$ so that $h\left(x_{\mathbf{y}_{\leq k}}^{i}\right)=y_{k+1}^{i}$ for all $0 \leq i \leq k$ and $0 \leq k \leq n$, where we denote

$\mathbf{y}_{\leq k}=\left(y_{1}^{0},\left(y_{2}^{0}, y_{2}^{1}\right), \ldots,\left(y_{k}^{0}, \ldots, y_{k}^{k-1}\right)\right), \quad x_{\mathbf{y}_{\leq k}}=\left(x_{\mathbf{y}_{\leq k}}^{0}, \ldots, x_{\mathbf{y}_{\leq k}}^{k}\right)$. We say that $\mathcal{H}$ has an infinite VCL tree if it has a VCL tree of depth $d=\infty$. 


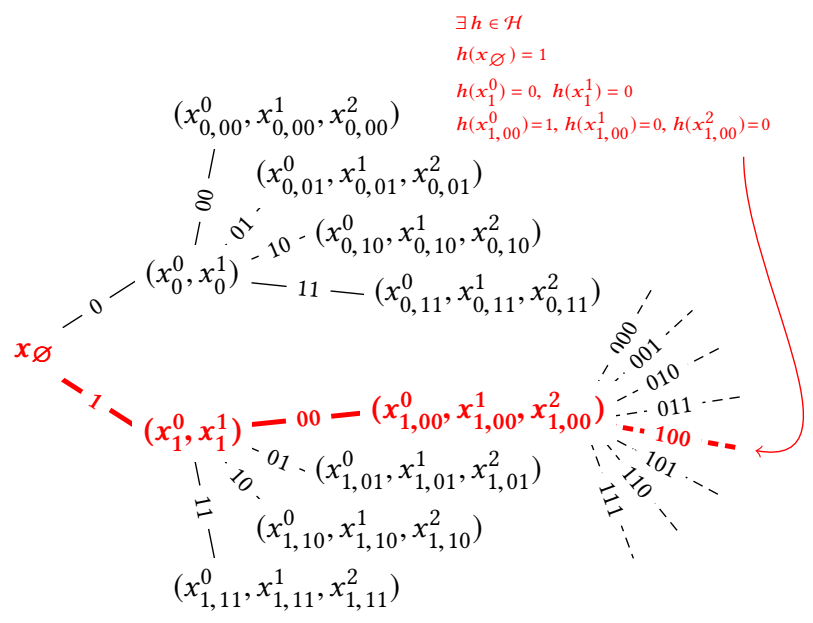

Figure 3: A VCL tree of depth 3. Every branch is consistent with a concept $h \in \mathcal{H}$. This is illustrated here for one of the branches. Due to lack of space, not all external edges are drawn.

A VCL tree resembles a Littlestone tree, except that each node in a VCL tree is labelled by a sequence of $k$ points, where $k$ is the depth of the node (in contrast, every node in a Littlestone tree is labelled by a single point). The branching factor at each node at depth $k$ of a VCL tree is thus $2^{k}$, rather than 2 as in a Littlestone tree. In the language of Vapnik-Chervonenkis theory, this means that along each path in the tree, we encounter shattered sets of size increasing with depth.

With these definitions in hand, we can state our second main result: a complete characterization of the optimal rate achievable for any given concept class $\mathcal{H}$.

Theorem 1.9. For every concept class $\mathcal{H}$ with $|\mathcal{H}| \geq 3$, the following hold:

- If $\mathcal{H}$ does not have an infinite Littlestone tree, then $\mathcal{H}$ is learnable with optimal rate $e^{-n}$

- If $\mathcal{H}$ has an infinite Littlestone tree but does not have an infinite VCL tree, then $\mathcal{H}$ is learnable with optimal rate $\frac{1}{n}$.

- If $\mathcal{H}$ has an infinite VCL tree, then $\mathcal{H}$ requires arbitrarily slow rates.

In particular, since Theorem 1.6 follows immediately from Theorem 1.9, the focus of this work will be to prove Theorem 1.9. The proof of this theorem, and many related results, are presented in the remainder of this paper.

\subsection{Technical Overview}

We next discuss some technical aspects in the derivation of the trichotomy. We also highlight key differences with the dichotomy of PAC learning theory.

1.5.1 Upper Bounds. In the uniform setting, the fact that every VC class is PAC learnable is witnessed by any algorithm that outputs an concept $h \in \mathcal{H}$ that is consistent with the input sample. This is known in the literature as the empirical risk minimization (ERM) principle and follows from the celebrated uniform convergence theorem of [26]. Moreover, any ERM algorithm achieves the optimal uniform learning rate, up to lower order factors.

In contrast, in the universal setting one has to carefully design the algorithms that achieve the optimal rates. In particular, here the optimal rates are not always achieved by general ERM methods: for example, there are classes where exponential rates are achievable, but where there exist ERM learners with arbitrarily slow rates (see Example 2.6 below). The learning algorithms we propose below are novel in the literature: they are based on the theory of infinite (GaleStewart) games, whose connection with learning theory appears to be new in this paper.

As was anticipated in the previous section, a basic building block of our learning algorithms is the solution of analogous problems in adversarial online learning. For example, as a first step towards a statistical learning algorithm that achieves exponential rates, we extend the mistake bound model of [18] to scenarios where it is possible to guarantee a finite number of mistakes for each realizable sequence, but without an a priori bound on the number of mistakes. We show this is possible precisely when $\mathcal{H}$ has no infinite Littlestone tree, in which case the resulting online learning algorithm is defined by the winning strategy of an associated GaleStewart game.

Unfortunately, while online learning algorithms may be applied directly to random training data, this does not in itself suffice to ensure good learning rates. The problem is that, although the online learning algorithm is guaranteed to make no mistakes after a finite number of rounds, in the statistical context this number of rounds is a random variable for which we have no control on the variance or tail behavior. We must therefore introduce additional steps to convert such online learning algorithms into statistical learning algorithms. In the case of exponential rates, this will be done by applying the online learning algorithm to several different batches of training examples, which must then be carefully aggregated to yield a classifier that achieves an exponential rate.

The case of linear rates presents additional complications. In this setting, the corresponding online learning algorithm does not eventually stop making mistakes: it is only guaranteed to eventually rule out a finite pattern of labels (which is feasible precisely when $\mathcal{H}$ has no infinite VCL tree). Once we have learned to rule out one pattern of labels for every data sequence of length $k$, the situation becomes essentially analogous to that of a VC class of dimension $k-1$. In particular, we can then apply the one-inclusion graph predictor of Haussler, Littlestone, and Warmuth [16] to classify subsequent data points with a linear rate. When applied to random data, however, both the time it takes for the online algorithm to learn to rule out a pattern, and the length $k$ of that pattern, are random. We must therefore again apply this technique to several different batches of training examples and combine the resulting classifiers with aggregation methods to obtain a statistical learning algorithm that achieves a linear rate.

1.5.2 Lower Bounds. The proofs of our lower bounds are also significantly more involved than those in PAC learning theory. In contrast to the uniform setting, we are required to produce a single data distribution $P$ for which the given learning algorithm has the claimed lower bound for infinitely many $n$. To this end, we will 
apply the probabilistic method by randomizing over both the choice of target labellings for the space, and the marginal distribution on $X$, coupling these two components of $P$.

1.5.3 Constructability and Measurability. There is a serious technical issue that arises in our theory that gives rise to surprisingly interesting mathematical questions. In order to apply the winning strategies of Gale-Stewart games to random data, we must ensure such strategies are measurable: if this is not the case, our theory may fail spectacularly (see the Appendix of the full paper [6]). However, nothing appears to be known in the literature about the measurability of Gale-Stewart strategies in nontrivial settings.

That measurability issues arise in learning theory is not surprising, of course; this is also the case in classical PAC learning $[5,20]$. Our basic measurability assumption (see the full paper [6]) is also the standard assumption made in this setting [10]. It turns out, however, that measurability issues in classical learning theory are essentially benign: the only issue that arises there is the measurability of the supremum of the empirical process over $\mathcal{H}$. This can be trivially verified in most practical situations without the need for an abstract theory: for example, measurability of the empirical process is trivial when $\mathcal{H}$ is countable, or when $\mathcal{H}$ can be pointwise approximated by a countable class. For these reasons, measurability issues in classical learning theory are often considered "a minor nuisance". The situation in this paper is completely different: it is entirely unclear a priori whether Gale-Stewart strategies are measurable even in apparently trivial cases, such as when $\mathcal{H}$ is countable.

We will prove the existence of measurable strategies for a general class of Gale-Stewart games that includes all the ones encountered in this paper. The solution of this problem exploits an interplay between the mathematical and algorithmic aspects of the problem. To construct a measurable strategy, we will explicitly define a strategy by means of a kind of greedy algorithm that aims to minimize in each step a value function that takes values in the ordinal numbers. This construction gives rise to unexpected new notions for learning theory: for example, we will show that the complexity of online learning is characterized by an ordinal notion of Littlestone dimension, which agrees with the classical notion when it is finite. To conclude the proof of measurability, we combine these insights with a deep result of descriptive set theory (the Kunen-Martin theorem) which shows that the Littlestone dimension of a measurable class $\mathcal{H}$ is always a countable ordinal.

\subsection{Related Work}

To conclude the introduction, we briefly review prior work on the subject of universal learning rates.

1.6.1 Universal Consistency. An extreme notion of learnability in the universal setting is universal consistency: a learning algorithm is universally consistent if $\mathbf{E}\left[\operatorname{er}\left(\hat{h}_{n}\right)\right] \rightarrow \inf _{h} \operatorname{er}(h)$ for every distribution $P$. The first proof that universally consistent learning is possible was provided by Stone [23], using local average estimators, such as based on k-nearest neighbor predictors, kernel rules, and histogram rules; see [9] for a thorough discussion of such results. One can also establish universal consistency of learning rules via the technique of structural risk minimization from Vapnik and Chervonenkis [27].
The most general results on universal consistency were recently established by Hanneke [14] and Hanneke, Kontorovich, Sabato, and Weiss [15], who proved the existence of universally consistent learning algorithms in any separable metric space. In fact, Hanneke, Kontorovich, Sabato, and Weiss [15] establish this for even more general spaces, called essentially separable, and prove that the latter property is actually necessary for universal consistency to be possible. An immediate implication of their result is that in such spaces $\mathcal{X}$, and choosing $\mathcal{H}$ to be the set of all measurable functions, there exists a learning algorithm with $\mathrm{E}\left[\operatorname{er}\left(\hat{h}_{n}\right)\right] \rightarrow 0$ for all realizable distributions $P$ (cf. Example 1.3). In particular, since we assume in this paper that $\mathcal{X}$ is Polish (i.e., separably metrizable), this result holds in our setting.

While these results establish that it is always possible to have $\mathrm{E}\left[\operatorname{er}\left(\hat{h}_{n}\right)\right] \rightarrow 0$ for all realizable $P$, there is a so-called no free lunch theorem showing that it is not generally possible to bound the rate of convergence: that is, the set $\mathcal{H}$ of all measurable functions requires arbitrarily slow rates [9]. The proof of this result also extends to more general concept classes: the only property of $\mathcal{H}$ that was used in the proof is that it finitely shatters some countably infinite subset of $\mathcal{X}$, that is, there exists $\mathcal{X}^{\prime}=\left\{x_{1}, x_{2}, \ldots\right\} \subseteq \mathcal{X}$ such that, for every $n \in \mathbb{N}$ and $y_{1}, \ldots, y_{n} \in\{0,1\}$, there is $h \in \mathcal{H}$ with $h\left(x_{i}\right)=y_{i}$ for every $i \leq n$. It is natural to wonder whether the existence of such a countable finitely shattered set $X^{\prime}$ is also necessary for $\mathcal{H}$ to require arbitrarily slow rates. Our main result settles this question in the negative. Indeed, Theorem 1.9 states that the existence of an infinite VCL tree is both necessary and sufficient for a concept class $\mathcal{H}$ to require arbitrarily slow rates; but it is possible for a class $\mathcal{H}$ to have an infinite VCL tree while it does not finitely shatter any countable set $\mathcal{X}^{\prime}$ (see Example 2.8 below).

1.6.2 Exponential versus Linear Rates. The distinction between exponential and linear rates has been studied by Schuurmans [22] in some special cases. Specifically, Schuurmans [22] studied classes $\mathcal{H}$ that are concept chains, meaning that every $h, h^{\prime} \in \mathcal{H}$ have either $h \leq h^{\prime}$ everywhere or $h^{\prime} \leq h$ everywhere. For instance, threshold classifiers on the real line (Example 1.2) are a simple example of a concept chain.

Since any concept chain $\mathcal{H}$ must have VC dimension at most 1 , the optimal rates can never be slower than linear [16]. However, Schuurmans [22] found that some concept chains are universally learnable at an exponential rate, and gave a precise characterization of when this is the case. Specifically, he established that a concept chain $\mathcal{H}$ is learnable at an exponential rate if and only if $\mathcal{H}$ is nowhere dense, meaning that there is no infinite subset $\mathcal{H}^{\prime} \subseteq \mathcal{H}$ such that, for every distinct $h_{1}, h_{2} \in \mathcal{H}^{\prime}$ with $h_{1} \leq h_{2}$ everywhere, $\exists h_{3} \in \mathcal{H}^{\prime} \backslash\left\{h_{1}, h_{2}\right\}$ with $h_{1} \leq h_{3} \leq h_{2}$ everywhere. He also showed that concept chains $\mathcal{H}$ failing this property (i.e., that are somewhere dense) are not learnable at rate faster than $n^{-(1+\varepsilon)}$ (for any $\varepsilon>0$ ); under further special conditions, he sharpened this lower bound to a strictly linear rate $n^{-1}$.

It is not difficult to see that for concept chain classes, the property of being somewhere dense precisely corresponds to the property of having an infinite Littlestone tree, where the above set $\mathcal{H}^{\prime}$ corresponds to the set of classifiers involved in the definition of the infinite Littlestone tree. Theorem 1.9 therefore recovers the result 
of Schuurmans [22] as a very special case, and sharpens his $n^{-(1+\epsilon)}$ general lower bound to a strict linear rate $n^{-1}$.

Schuurmans [22] also posed the question of whether his analysis can be extended beyond concept chains: that is, whether there is a general characterization of which classes $\mathcal{H}$ are learnable at an exponential rate, versus which classes are not learnable at faster than a linear rate. This question is completely settled by the main results of this paper.

1.6.3 Classes with Matching Universal and Uniform Rates. Antos and Lugosi [1] showed that there exist concept classes for which no improvement on the PAC learning rate is possible in the universal setting. More precisely, they showed that, for any $d \in \mathbb{N}$, there exists a concept class $\mathcal{H}$ of VC dimension $d$ such that, for any learning algorithm $\hat{h}_{n}$, there exists a realizable distribution $P$ for which $\mathrm{E}\left[\operatorname{er}\left(\hat{h}_{n}\right)\right] \geq \frac{c d}{n}$ for infinitely many $n$, where the numerical constant $c$ can be made arbitrarily close to $\frac{1}{2}$. This shows that universal learning rates for some classes tightly match their minimax rates up to a numerical constant factor.

1.6.4 Active Learning. Universal learning rates have also been considered in the context of active learning, under the names true sample complexity or unverifiable sample complexity [3, 13, 28]. Active learning is a variant of supervised learning, where the learning algorithm observes only the sequence $X_{1}, X_{2}, \ldots$ of unlabeled examples, and may select which examples $X_{i}$ to query (which reveals their labels $Y_{i}$ ); this happens sequentially, so that the learner observes the response to a query before selecting its next query point. In this setting, one is interested in characterizing the rate of convergence of $\mathrm{E}\left[\operatorname{er}\left(\hat{h}_{n}\right)\right]$ where $n$ is the number of queries (i.e., the number of labels observed) as opposed to the sample size.

Hanneke $[12,13]$ showed that for any VC class $\mathcal{H}$, there is an active learning algorithm $\hat{h}_{n}$ such that, for every realizable distribution $P, \mathbf{E}\left[\operatorname{er}\left(\hat{h}_{n}\right)\right]=o\left(\frac{1}{n}\right)$. Note that such a result is certainly not achievable by passive learning algorithms (i.e., the type of learning algorithms discussed in the present work), given the results of Schuurmans [22] and Antos and Lugosi [1]. The latter also follows from the results of this paper by Example 2.2 below.

1.6.5 Nonuniform Learning. Denote by $\operatorname{RE}(h)$ the family of distributions $P$ such that $\operatorname{er}(h)=0$ for a given classifier $h \in H$. Benedek and Itai [4] considered a partial relaxation of the PAC model, called nonuniform learning, in which the learning rate may depend on $h \in \mathcal{H}$ but is still uniform over $P \in \operatorname{RE}(h)$. This setting intermediate between the PAC setting (where the rate may depend only on $n$ ) and the universal learning setting (where the rate may depend fully on $P$ ). A concept class $\mathcal{H}$ is said to be learnable in the nonuniform learning setting if there exists a learning algorithm $\hat{h}_{n}$ such that $\sup _{P \in \operatorname{RE}(h)} \mathrm{E}\left[\operatorname{er}\left(\hat{h}_{n}\right)\right] \rightarrow 0$ as $n \rightarrow \infty$ for every $h \in \mathcal{H}$.

Benedek and Itai [4] proved that a concept class $\mathcal{H}$ is learnable in the nonuniform learning model if and only if $\mathcal{H}$ is a countable union of VC classes. In Example 2.7 below, we show that there exist classes $\mathcal{H}$ that are universally learnable, even at an exponential rate, but which are not learnable in the nonuniform learning setting. It is also easy to observe that there exist classes $\mathcal{H}$ that are countable unions of VC classes (hence nonuniformly learnable) which have an infinite VCL tree (and thus require arbitrarily slow universal

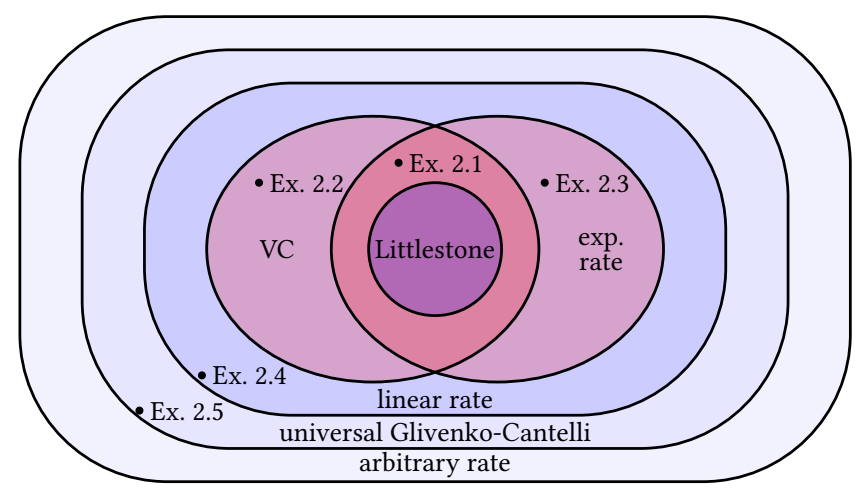

Figure 4: A Venn diagram depicting the trichotomy and its relation with uniform and universal learnability. While the focus here is on statistical learning, note that this diagram also captures the distinction between uniform and universal online learning; see the full paper [6].

learning rates). The universal and nonuniform learning models are therefore incomparable.

\section{EXAMPLES}

In Section 1.3, we introduced three basic examples that illustrate the three possible universal learning rates. In this section we provide further examples. The main aim of this section is to illustrate important distinctions with the uniform setting and other basic concepts in learning theory, which are illustrated schematically in Figure 4.

\subsection{Universal Learning versus PAC Learning}

We begin by giving four examples that illustrate that the classical PAC learning model (which is characterized by finite VC dimension) is not comparable to the universal learning model.

Example 2.1 (VC with exponential rate). Consider the class $\mathcal{H} \subseteq$ $\{0,1\}^{\mathbb{N}}$ of all threshold functions $h_{t}(x)=\mathbf{1}_{x \geq t}$ where $t \in \mathbb{N}$. This is a VC class (its VC dimension is 1), which is learnable at an exponential rate (it does not have an infinite Littlestone tree). Note, however, that this class has unbounded Littlestone dimension (it shatters Littlestone trees of arbitrary finite depths), so that it does not admit an online learning algorithm that makes a uniformly bounded number of mistakes.

Example 2.2 (VC with linear rate). Consider the class $\mathcal{H} \subseteq\{0,1\}^{\mathbb{R}}$ of all threshold functions $h_{t}(x)=1_{x \geq t}$, where $t \in \mathbb{R}$. This is a VC class (its VC dimension is 1) that is not learnable at an exponential rate (it has an infinite Littlestone tree). Thus the optimal rate is linear.

Example 2.3 (Exponential rate but not VC). Let $\mathcal{X}=\cup_{k} \mathcal{X}_{k}$ be the disjoint union of finite sets $\left|X_{k}\right|=k$. For each $k$, let $\mathcal{H}_{k}=$ $\left\{1_{S}: S \subseteq \mathcal{X}_{k}\right\}$, and consider the concept class $\mathcal{H}=\cup_{k} \mathcal{H}_{k}$. This class has an unbounded VC dimension, yet is universally learnable at an exponential rate. To establish the latter, it suffices to prove that $\mathcal{H}$ does not have an infinite Littlestone tree. Indeed, once we fix any root label $x \in \mathcal{X}_{k}$ of a Littlestone tree, only $h \in \mathcal{H}_{k}$ 
can satisfy $h(x)=1$, and so the hypotheses consistent with the subtree corresponding to $h(x)=1$ form a finite class. This subtree can therefore have only finitely many leaves, contradicting the existence of an infinite Littlestone tree.

Example 2.4 (Linear rate but not VC). Consider the disjoint union of the classes of Examples 2.2 and 2.3: that is, $\mathcal{X}$ is the disjoint union of $\mathbb{R}$ and finite sets $\mathcal{X}_{k}$ with $\left|\mathcal{X}_{k}\right|=k$, and $\mathcal{H}$ is the union of the class of all threshold functions on $\mathbb{R}$ and the classes $\mathcal{H}_{k}=\left\{\mathbf{1}_{S}: S \subseteq\right.$ $\left.X_{k}\right\}$. This class has an unbounded VC dimension, yet is universally learnable at a linear rate. To establish the latter, it suffices to note that $\mathcal{H}$ has an infinite Littlestone tree as in Example 2.2, but $\mathcal{H}$ cannot have an infinite VCL tree. Indeed, once we fix any root label $x \in \mathcal{X}$, the class $\{h \in \mathcal{H}: h(x)=1\}$ has finite VC dimension, and thus the corresponding subtree of the VCL tree must be finite.

\subsection{Universal Learning Algorithms versus ERM}

The aim of the next two examples is to shed some light on the type of algorithms that can give rise to optimal universal learning rates. Recall that in the PAC model, a concept class is learnable if and only if it can be learned by any ERM (empirical risk minimization) algorithm. The following examples will show that the ERM principle cannot explain the achievable universal learning rates; the algorithms developed in this paper are thus necessarily of a different nature.

An ERM algorithm is any learning rule that outputs a concept in $\mathcal{H}$ that minimizes the empirical error. There may in fact be many such hypotheses, and thus there are many inequivalent ERM algorithms. Learnability by means of a general ERM algorithm is equivalent to the Glivenko-Cantelli property: that is, that the empirical errors of all $h \in \mathcal{H}$ converge simultaneously to the corresponding population errors as $n \rightarrow \infty$. The Glivenko-Cantelli property has a uniform variant, in which the convergence rate is uniform over all data distributions $P$; this property is equivalent to PAC learnability and is characterized by VC dimension [26]. It also has a universal variant, where the convergence holds for every $P$ but with distribution-dependent rate; the latter is equivalent to the universal consistency of a general ERM algorithm. A combinatorial characterization of the universal Glivenko-Cantelli property is given by van Handel [25].

The following example shows that even if a concept class is universally learnable by a general ERM algorithm, this need not yield any control on the learning rate. This is in contrast to the PAC setting, where learnability by means of ERM always implies a linear learning rate.

Example 2.5 (Arbitrarily slow rates but learnable by any ERM). Let $\mathcal{X}=\mathbb{N}$ and let $\mathcal{H}$ be the class of all classifiers on $\mathcal{X}$. This class has an infinite VCL tree and thus requires arbitrarily slow rates; but $\mathcal{H}$ is a universal Glivenko-Cantelli class and thus any ERM algorithm is universally consistent.

In contrast, the next example shows that there are are scenarios where extremely fast universal learning is achievable, but where a general ERM algorithm can give rise to arbitrarily slow rates.

Example 2.6 (Exponential rate achivable but general ERM arbitrarily slow). Let $X=\bigcup_{i \in \mathbb{N}} X_{i}$ be the disjoint union of finite sets with $\left|\mathcal{X}_{i}\right|=2^{i}$. For each $i \in \mathbb{N}$, let

$$
\mathcal{H}_{i}=\left\{\mathbf{1}_{I}: I \subseteq \mathcal{X}_{i},|I| \geq 2^{i-1}\right\},
$$

and consider the concept class $\mathcal{H}=\bigcup_{i \in \mathbb{N}} \mathcal{H}_{i}$. It follows exactly as in Example 2.3 that $\mathcal{H}$ has no infinite Littlestone tree, so that it is universally learnable at an exponential rate.

We claim there exists, for any rate function $R(n) \rightarrow 0$, an ERM algorithm that achieves rate slower than $R$. In the following, we fix any such $R$, as well as strictly increasing sequences $\left\{n_{t}\right\}$ and $\left\{i_{t}\right\}$ satisfying the following: letting $p_{t}=\frac{2^{i_{t}-2}}{n_{t}}$, it holds that $p_{t}$ is decreasing, $\sum_{t=1}^{\infty} p_{t} \leq 1$, and $p_{t} \geq 4 R\left(n_{t}\right)$. The reader may verify that such sequences can be constructed by induction on $t$.

Now consider any ERM with the following property: if the input data $\left(X_{1}, Y_{1}\right), \ldots,\left(X_{n}, Y_{n}\right)$ is such that $Y_{i}=0$ for all $i$, then the algorithm outputs $\hat{h}_{n} \in \mathcal{H}_{i_{n}}$ with

$T_{n}=\min \left\{t:\right.$ there exists $h \in \mathcal{H}_{i_{t}}$ s.t. $\left.h\left(X_{1}\right)=\cdots=h\left(X_{n}\right)=0\right\}$.

We claim that such ERM perform poorly on the data distribution $P$ defined by

$$
P\{(x, 0)\}=2^{-i_{t}} p_{t} \quad \text { for all } x \in \mathcal{X}_{i_{t}}, t \in \mathbb{N},
$$

where we set $P\left\{\left(x^{\prime}, 0\right)\right\}=1-\sum_{t=1}^{\infty} p_{t}$ for some arbitrary choice of $x^{\prime} \notin \cup_{t \in \mathbb{N}} \mathcal{X}_{i_{t}}$. Note that $P$ is realizable, as $\inf _{i} \operatorname{er}\left(h_{i}\right) \leq \inf _{i} P\{(x, y)$ : $\left.x \in \mathcal{X}_{i}\right\}=0$ for any $h_{i} \in \mathcal{H}_{i}$.

It remains to show that $\mathbf{E}\left[\operatorname{er}\left(\hat{h}_{n}\right)\right] \geq R(n)$ for infinitely many $n$. To this end, note that by Markov's inequality, there is a probability at least $1 / 2$ that the number of $\left(X_{1}, Y_{1}\right), \ldots,\left(X_{n_{t}}, Y_{n_{t}}\right)$ such that $X_{j} \in X_{i_{t}}$ is at most $2^{i_{t}-1}$. On this event, we must have $T_{n} \leq t$, so that

$$
\operatorname{er}\left(\hat{h}_{n_{t}}\right) \geq \frac{1}{2} P\left\{(x, 0): x \in \mathcal{X}_{i_{T n}}\right\} \geq \frac{p_{t}}{2} \geq 2 R\left(n_{t}\right) .
$$

Thus we have shown that $\mathrm{E}\left[\operatorname{er}\left(\hat{h}_{n_{t}}\right)\right] \geq R\left(n_{t}\right)$ for all $t \in \mathbb{N}$.

\subsection{Universal Learning versus Other Learning Models}

The nonuniform learning model of Benedek and Itai [4] is intermediate between universal and PAC learning; see Section 1.6.5. Our next example shows that a concept class may be not even learnable in the nonuniform sense, while exhibiting the fastest rate of uniform learning.

Example 2.7 (Exponential rate but not nonuniformly learnable). The following class can be learned at an exponential rate, yet it cannot be presented as a countable union of VC classes (and hence it is not learnable in the nonuniform setting by 4$)$ :

$$
\mathcal{X}=\{S \subset \mathbb{R}:|S|<\infty\}, \quad \mathcal{H}=\left\{h_{y}: y \in \mathbb{R}\right\},
$$

where $h_{y}(S)=\mathbf{1}_{y \in S}$. We first claim that $\mathcal{H}$ has no infinite Littlestone tree: indeed, once we fix a root label $S \in \mathcal{X}$ of a Littlestone tree, the class $\{h \in \mathcal{H}: h(S)=1\}$ is finite, so the corresponding subtree must be finite. Thus $\mathcal{H}$ is universally learnable at an exponential rate.

On the other hand, suppose that $\mathcal{H}$ were a countable union of VC classes. Then one element of this countable union must contain infinitely many hypotheses (as $\mathbb{R}$ is uncountable). This is a contradiction, as any infinite subset $\left\{h_{y}: y \in I\right\} \subseteq \mathcal{H}$ with $I \subseteq \mathbb{R}$, $|I|=\infty$ has unbounded VC dimension (as its dual class is the class of all finite subsets of $I$ ). 
Our next example is concerned with the characterization of arbitrarily slow rates. As we discussed in Section 1.6.1, a no free lunch theorem of Devroye, Györfi, and Lugosi [9] shows that a sufficient condition for a class $\mathcal{H}$ to require arbitrarily slow rates is that there exists an infinite set $\mathcal{X}^{\prime} \subseteq \mathcal{X}$ finitely shattered by $\mathcal{H}$ : that is, there exists $\mathcal{X}^{\prime}=\left\{x_{1}, x_{2}, \ldots\right\} \subseteq \mathcal{X}$ such that, for every $n \in \mathbb{N}$ and $y_{1}, \ldots, y_{n} \in\{0,1\}$, there is $h \in \mathcal{H}$ with $h\left(x_{i}\right)=y_{i}$ for every $i \leq n$. Since our Theorem 1.9 indicates that existence of an infinite VCL tree is both sufficient and necessary, it is natural to ask how these two conditions relate to each other. It is easy to see that the existence of a finitely shattered infinite set $\mathcal{X}^{\prime}$ implies the existence of an infinite VCL tree. However, the following example shows that the opposite is not true: that is, there exist classes $\mathcal{H}$ with an infinite VCL tree that do not finitely shatter an infinite set $\mathcal{X}^{\prime}$. Thus, these conditions are not equivalent, and our Theorem 1.9 provides a strictly weaker condition sufficient for $\mathcal{H}$ to require arbitrarily slow rates.

Example 2.8 (No finitely shattered infinite set, but requires arbitrarily slow rates). Consider a countable space $\mathcal{X}$ that is itself structured into nodes of a VCL tree: that is,

$\mathcal{X}=\left\{x_{\mathbf{u}}^{i}: k \in \mathbb{N} \cup\{0\}, i \in\{0, \ldots, k\}, \mathbf{u} \in\{0,1\}^{1} \times\{0,1\}^{2} \times \cdots \times\{0,1\}^{k}\right\}$, where each $x_{\mathbf{u}}^{i}$ is a distinct point. Then for each

$\mathbf{y}=\left(y_{1}^{0},\left(y_{2}^{0}, y_{2}^{1}\right), \ldots,\left(y_{k}^{0}, \ldots, y_{k}^{k-1}\right), \ldots\right) \in\{0,1\}^{1} \times\{0,1\}^{2} \times \cdots$, define $h_{\mathrm{y}}$ such that every $k \in \mathbb{N} \cup\{0\}$ and $i \in\{0, \ldots, k\}$ has $h_{\mathbf{y}}\left(x_{\mathbf{y}_{\leq k}}^{i}\right)=y_{k+1}^{i}$, and every $x \in \mathcal{X} \backslash\left\{x_{\mathbf{y}_{\leq k}}^{i}: k \in \mathbb{N} \cup\{0\}, i \in\right.$ $\{0, \ldots, k\}\}$ has $h_{\mathrm{y}}(x)=0$. Then define

$$
\mathcal{H}=\left\{h_{\mathrm{y}}: \mathrm{y} \in\{0,1\}^{1} \times\{0,1\}^{2} \times \cdots\right\} .
$$

By construction, this class $\mathcal{H}$ has an infinite VCL tree. However, any set $S \subset \mathcal{X}$ of size at least 2 which is shattered by $\mathcal{H}$ must be contained within a single node of the tree. In particular, since any countable set $\mathcal{X}^{\prime}=\left\{x_{1}^{\prime}, x_{2}^{\prime}, \ldots\right\} \subseteq \mathcal{X}$ necessarily contains points $x_{i}^{\prime}, x_{j}^{\prime}$ existing in different nodes of the tree, the set $\left\{x_{1}^{\prime}, \ldots, x_{\max \{i, j\}}^{\prime}\right\}$ is not shattered by $\mathcal{H}$, so that $X^{\prime}$ is not finitely shattered by $\mathcal{H}$.

\subsection{Geometric Examples}

The previous examples were designed to illustrate the key features of the results of this paper in comparison with other learning models; however, these examples may be viewed as somewhat artificial. To conclude this section, we give two examples of "natural" geometric concept classes that are universally learnable with exponential rate. This suggests that our theory has direct implications for learning scenarios of the kind that may arise in applications.

Example 2.9 (Nonlinear manifolds). Various practical learning problems are naturally expressed by concepts that indicate whether the data lie on a manifold. The following construction provides one simple way to model classes of nonlinear manifolds. Let the domain $\mathcal{X}$ be any Polish space, and fix a measurable function $g: \mathcal{X} \rightarrow \mathbb{R}^{d}$ with $d<\infty$. For a given $k<\infty$, consider the concept class

$$
\mathcal{H}=\left\{\mathbf{1}_{A g=0}: A \in \mathbb{R}^{k \times d}\right\} .
$$

The coordinate functions $g_{1}, \ldots, g_{d}$ describe the nonlinear features of the class. For example, if $\mathcal{X}=\mathbb{C}^{n}$ and $g_{j}$ are polynomials, this model can describe any class of affine algebraic varieties.
We claim that $\mathcal{H}$ is universally learnable at exponential rate. It suffices to show that, in fact, $\mathcal{H}$ has finite Littlestone dimension. To see why, fix any Littlestone tree, and consider its branch $x_{\varnothing}, x_{1}, x_{11}, \ldots$; for simplicity, we will denote these points in this example as $x^{0}, x^{1}, x^{2}, \ldots$. Define

$$
V_{j}=\left\{A \in \mathbb{R}^{k \times d}: A g\left(x^{i}\right)=0 \text { for } i=0, \ldots, j\right\} .
$$

Each $V_{j}$ is a finite-dimensional linear space. Now note that if $V_{j}=$ $V_{j-1}$, then all $h \in \mathcal{H}$ such that $h\left(x^{i}\right)=1, i=1, \ldots, j-1$ satisfy $h\left(x^{j}\right)=1$; but this is impossible, as the definition of a Littlestone tree requires the existence of $h \in \mathcal{H}$ such that $h\left(x^{i}\right)=1, i=1, \ldots, j-1$ and $h\left(x^{j}\right)=0$. Thus the dimension of $V_{j}$ must decrease strictly in $j$, so the branch $x_{\varnothing}, x_{1}, x_{11}, \ldots$ must be finite.

Example 2.10 (Positive halfspaces on $\mathbb{N}^{d}$ ). It is a classical fact that the class of halfspaces on $\mathbb{R}^{d}$ has finite VC dimension, and it is easy to see this class has an infinite Littlestone tree. Thus the PAC rate cannot be improved in this setting. The aim of this example is to show that the situation is quite different if one considers positive halfspaces on a lattice $\mathbb{N}^{d}$ : such a class is universally learnable with exponential rate. This may be viewed as an extension of Example 2.1, which illustrates that some geometric classes on discrete spaces can be universally learned at a much faster rate than geometric classes on continuous spaces (a phenomenon not captured by the PAC model).

More precisely, let $\mathcal{X}=\mathbb{N}^{d}$ for some $d \in \mathbb{N}$, and let $\mathcal{H}$ be the class of positive halfspaces:

$$
\mathcal{H}=\left\{\mathbb{1}_{\mathbf{w} \cdot \mathbf{x}-b \geq 0}:(\mathbf{w}, b) \in(0, \infty)^{d+1}\right\} .
$$

We will argue that $\mathcal{H}$ is universally learnable at an exponential rate by constructing an explicit learning algorithm guaranteeing a finite number of mistakes for every realizable data sequence. As is argued in the full paper [6], the existence of such an algorithm immediately implies $\mathcal{H}$ does not have an infinite Littlestone tree. Moreover, we also show in the full paper [6] that such an algorithm can be converted into a learning algorithm achieving exponential rates for all realizable distributions $P$.

Let $S_{n} \in(\mathcal{X} \times\{0,1\})^{n}$ be any data set consistent with some $h \in \mathcal{H}$. If every $\left(x_{i}, y_{i}\right) \in S_{n}$ has $y_{i}=0$, let $\hat{h}_{n}(x)=0$ for all $x \in \mathcal{X}$.

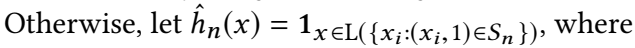

$\mathrm{L}\left(\left\{z_{1}, \ldots, z_{t}\right\}\right)=\left\{z^{\prime}+\sum_{i \leq t} \alpha_{i} z_{i}: \alpha_{i} \in[0,1], \sum_{i \leq t} \alpha_{i}=1, z^{\prime} \in[0, \infty)^{d}\right\}$

for any $t \in \mathbb{N}$ and $z_{1}, \ldots, z_{t} \in \mathcal{X} . \mathrm{L}\left(\left\{z_{1}, \ldots, z_{t}\right\}\right)$ is the smallest region containing the convex hull of $z_{1}, \ldots, z_{t}$ for which the indicator of the region is non-decreasing in every dimension.

Now consider any sequence $\left\{\left(x_{i}, y_{i}\right)\right\}_{i \in \mathbb{N}}$ in $X \times\{0,1\}$ such that for each $n \in \mathbb{N}$, letting $S_{n}=\left\{\left(x_{i}, y_{i}\right)\right\}_{i=1}^{n}$, there exists $h_{n}^{*} \in \mathcal{H}$ with $h_{n}^{*}\left(x_{i}\right)=y_{i}$ for all $i \leq n$. Since $\left\{x: h_{n+1}^{*}(x)=1\right\}$ is convex, and $h_{n+1}^{*}(x)$ is non-decreasing in every dimension, we have $\hat{h}_{n} \leq h_{n+1}^{*}$. This implies that any $n \in \mathbb{N}$ with $\hat{h}_{n}\left(x_{n+1}\right) \neq y_{n+1}$ must have $y_{n+1}=1$ and $\hat{h}_{n}\left(x_{n+1}\right)=0$. Therefore, by the definition of $\mathrm{L}(\cdot)$, the following must hold for any $n$ with $\hat{h}_{n}\left(x_{n+1}\right) \neq y_{n+1}$ : for every $i \leq n$ such that $y_{i}=1$, there exists a coordinate $1 \leq j \leq d$ such that $\left(x_{n+1}\right)_{j}<\left(x_{i}\right)_{j}$.

Now suppose, for the sake of obtaining a contradiction, that there is an increasing infinite sequence $\left\{n_{t}\right\}_{t \in \mathbb{N}}$ such that $\hat{h}_{n_{t}}\left(x_{n_{t}+1}\right) \neq$ 
$y_{n_{t}+1}$, and consider a coloring of the infinite complete graph with vertices $\left\{x_{n_{t}+1}\right\}_{t \in \mathbb{N}}$ where every edge $\left\{x_{n_{t}+1}, x_{n_{t^{\prime}+1}}\right\}$ with $t<t^{\prime}$ is colored with a value $\min \left\{j:\left(x_{n_{t^{\prime}}+1}\right)_{j}<\left(x_{n_{t}+1}\right)_{j}\right\}$. Then the infinite Ramsey theorem implies there exists an infinite monochromatic clique: that is, a value $j \leq d$ and an infinite subsequence $\left\{n_{t_{i}}\right\}$ with $\left(x_{n_{t_{i}}+1}\right)_{j}$ strictly decreasing in $i$. This is a contradiction, since clearly any strictly decreasing sequence $\left(x_{n_{t_{i}}+1}\right)_{j}$ maintaining $x_{n_{t_{i}}+1} \in \mathcal{X}$ can be of length at most $\left(x_{n_{t_{1}}+1}\right)_{j}$, which is finite. Therefore, the learning algorithm $\hat{h}_{n}$ makes at most a finite number of mistakes on any such sequence $\left\{\left(x_{i}, y_{i}\right)\right\}_{i \in \mathbb{N}}$. Let us note, however, that there can be no uniform bound on the number of mistakes (independent of the specific sequence $\left\{\left(x_{i}, y_{i}\right)\right\}_{i \in \mathbb{N}}$ ), since the Littlestone dimension of $\mathcal{H}$ is infinite.

\section{REFERENCES}

[1] A. Antos and G. Lugosi. 1998. Strong Minimax Lower Bounds for Learning Machine Learning 30 (1998), 31-56.

[2] J.-Y. Audibert and A. B. Tsybakov. 2007. Fast Learning Rates for Plug-in Classifiers. The Annals of Statistics 35, 2 (2007), 608-633.

[3] M.-F. Balcan, S. Hanneke, and J. Wortman Vaughan. 2010. The True Sample Complexity of Active Learning. Machine Learning 80, 2-3 (2010), 111-139.

[4] G. M. Benedek and A. Itai. 1994. Nonuniform Learnability. F. Comput. System Sci. 48 (1994), 311-323.

[5] A. Blumer, A. Ehrenfeucht, D. Haussler, and M. Warmuth. 1989. Learnability and the Vapnik-Chervonenkis Dimension. Journal of the Association for Computing Machinery 36, 4 (1989), 929-965.

[6] Olivier Bousquet, Steve Hanneke, Shay Moran, Ramon van Handel, and Amir Yehudayoff. 2020. A Theory of Universal Learning. CoRR abs/2011.04483 (2020). arXiv:2011.04483 https://arxiv.org/abs/2011.04483

[7] D. Cohn and G. Tesauro. 1990. Can Neural Networks Do Better than the VapnikChervonenkis Bounds?. In Advances in Neural Information Processing Systems.

[8] D. Cohn and G. Tesauro. 1992. How Tight are the Vapnik-Chervonenkis Bounds? Neural Computation 4, 2 (1992), 249-269.

[9] L. Devroye, L. Györfi, and G. Lugosi. 1996. A Probabilistic Theory of Pattern Recognition. Springer-Verlag New York, Inc.

[10] R. M. Dudley. 2014. Uniform central limit theorems (second ed.). Cambridge Studies in Advanced Mathematics, Vol. 142. Cambridge University Press, New York. xii+472 pages.

[11] A. Ehrenfeucht, D. Haussler, M. Kearns, and L. Valiant. 1989. A General Lower Bound on the Number of Examples Needed for Learning. Information and Computation 82, 3 (1989), 247-261.
[12] S. Hanneke. 2009. Theoretical Foundations of Active Learning. Ph.D. Dissertation. Machine Learning Department, School of Computer Science, Carnegie Mellon University.

[13] S. Hanneke. 2012. Activized Learning: Transforming Passive to Active with Improved Label Complexity. Fournal of Machine Learning Research 13, 5 (2012), 1469-1587.

[14] S. Hanneke. 2017. Learning Whenever Learning is Possible: Universal Learning under General Stochastic Processes. arXiv:1706.01418 (2017).

[15] S. Hanneke, A. Kontorovich, S. Sabato, and R. Weiss. 2019. Universal Bayes Consistency in Metric Spaces. arXiv:1705.08184 (2019).

[16] D. Haussler, N. Littlestone, and M. Warmuth. 1994. Predicting $\{0,1\}$-Functions on Randomly Drawn Points. Information and Computation 115, 2 (1994), 248-292.

[17] V. Koltchinskii and O. Beznosova. 2005. Exponential Convergence Rates in Classification. In Learning Theory, 18th Annual Conference on Learning Theory, COLT 2005, Bertinoro, Italy, June 27-30, 2005, Proceedings (Lecture Notes in Computer Science, Vol. 3559), Peter Auer and Ron Meir (Eds.). Springer, 295-307. https://doi.org/10.1007/11503415_20

[18] N. Littlestone. 1988. Learning quickly when irrelevant attributes abound: A new linear-threshold algorithm. Machine Learning 2 (1988), 285-318.

[19] A. Nitanda and T. Suzuki. 2019. Stochastic Gradient Descent with Exponential Convergence Rates of Expected Classification Errors. In AISTATS (Proceedings of Machine Learning Research, Vol. 89). PMLR, 1417-1426.

[20] V. Pestov. 2011. PAC learnability versus VC dimension: A footnote to a basic result of statistical learning. In The 2011 International foint Conference on Neural Networks. 1141-1145. https://doi.org/10.1109/IJCNN.2011.6033352

[21] L. Pillaud-Vivien, A. Rudi, and F. Bach. 2018. Exponential Convergence of Testing Error for Stochastic Gradient Methods. In Conference On Learning Theory, COLT 2018, Stockholm, Sweden, 6-9 July 2018 (Proceedings of Machine Learning Research, Vol. 75), Sébastien Bubeck, Vianney Perchet, and Philippe Rigollet (Eds.). PMLR, 250-296. http://proceedings.mlr.press/v75/pillaud-vivien18a.html

[22] D. Schuurmans. 1997. Characterizing Rational Versus Exponential Learning Curves. 7. Comput. System Sci. 55, 1 (1997), 140-160.

[23] C. J. Stone. 1977. Consistent Nonparametric Regression. The Annals of Statistics (1977), 595-620.

[24] L. G. Valiant. 1984. A Theory of the Learnable. Commun. ACM 27, 11 (Nov. 1984), 1134-1142.

[25] R. van Handel. 2013. The Universal Glivenko-Cantelli Property. Probability and Related Fields 155 (2013), 911-934.

[26] V. Vapnik and A. Chervonenkis. 1971. On the uniform convergence of relative frequencies of events to their probabilities. Theory of Probability and its Applications 16, 2 (1971), 264-280.

[27] V. Vapnik and A. Chervonenkis. 1974. Theory of Pattern Recognition. Nauka, Moscow.

[28] L. Yang and S. Hanneke. 2013. Activized Learning with Uniform Classification Noise. In Proceedings of the $30^{\text {th }}$ International Conference on Machine Learning. 